\title{
ARTICLE OPEN Photorealistic modelling of metals from first principles
}

\author{
Gianluca Prandini ${ }^{1}$, Gian-Marco Rignanese $\mathbb{D D}^{2}$ and Nicola Marzari ${ }^{1 *}$
}

The colours of metals have attracted the attention of humanity since ancient times, and coloured metals, in particular gold compounds, have been employed for tools and objects symbolizing the aesthetics of power. In this work, we develop a comprehensive framework to obtain the reflectivity and colour of metals, and show that the trends in optical properties and the colours can be predicted by straightforward first-principles techniques based on standard approximations. We apply this to predict reflectivity and colour of several elemental metals and of different types of metallic compounds (intermetallics, solid solutions and heterogeneous alloys), considering mainly binary alloys based on noble metals. We validate the numerical approach through an extensive comparison with experimental data and the photorealistic rendering of known coloured metals.

npj Computational Materials (2019)5:129; https://doi.org/10.1038/s41524-019-0266-0

\section{INTRODUCTION}

Optical properties of metals are important for novel technological applications where the optical response needs to be engineered for specific purposes, such as for plasmonic devices (e.g. in spectrally selective coatings ${ }^{1,2}$ ), for optoelectronics devices (e.g. in ultra-thin films for transparent conductive electrodes ${ }^{3,4}$ ) and also for microscopy and optical data storage. ${ }^{5}$ Also, the colours of metals (which are related to the optical properties within the visible range of the electromagnetic spectrum) play a significant role in the jewellery industry, decoration and dentistry. For these applications, the most used materials are metallic alloys based on gold or other coinage or precious metals, such as silver, copper, palladium and platinum. In particular, gold and copper are the few elemental metals that show a characteristic colour, due to the presence of a drop in the reflectivity curve inside the visible range; reflectivities of nearly all other metals are instead generally high and flat for all visible frequencies, making them appear shiny and silvery white. Moreover, gold alloys and intermetallics are known to show a broad spectrum of colours (yellow, red, purple, to name a few), which can be tuned by varying the alloying elements and concentrations in the material. ${ }^{6}$ Since in the jewellery industry there is the demand, due to market and fashion trends, for new precious-metal alloys with specific colours, it is also of great interest there the search and identification of novel alloys with novel optical properties.

Generally speaking, the common route followed by researchers and manufacturers in order to identify any type of novel materials is through trial-and-error experiments, which, however, have the drawback to be time consuming and, if dealing with preciousmetal-based systems, expensive. In order to streamline this process, an alternative route that can help in guiding the search for new promising candidate systems is computational modelling, so that the physical properties under investigation are assessed through computer simulations rather than by real experiments. Here, we show and discuss how it is possible to perform photorealistic simulations of metals by means of first-principles methods and, as a consequence, predict the colour of novel metallic alloys. Previously published studies about first-principles simulation of optical properties of both elemental metals and alloys already point towards the feasibility of this approach.
Indeed, in 1988 Maksimov et al. ${ }^{7}$ computed the optical properties of several elemental metals, whereas, more recently, Werner et al. ${ }^{8}$ performed a similar study on 17 elemental metals; both studies found qualitative agreement with experimental results. For compounds, on the other hand, Blaber et al. ${ }^{9}$ calculated the optical properties of several intermetallic compounds, with a particular focus on alkali-noble intermetallics, for new possible candidates as plasmonic materials while, in another work, Keast et al. ${ }^{10}$ computed the density of states and dielectric function of gold intermetallics compounds and gold binary alloys. Regarding the simulation of specific coloured intermetallic compounds, the reflectivity and colour of the three well-known coloured gold intermetallics $\mathrm{AuAl}_{2}, \mathrm{AuGa}_{2}$ and $\mathrm{AuIn}_{2}$ was first computed in ref. ${ }^{11}$ and, afterwards, Keast et al. ${ }^{12}$ studied the influence of alloying concentrations on the reflectivity and colour of the intermetallic $\mathrm{AuAl}_{2}$ by considering the ternary compounds having the $\mathrm{Au}_{1}$ ${ }_{-}{ }_{x} \mathrm{Pt}_{x} \mathrm{Al}_{2}$ composition, with $x=0.0,0.5,0.75,1.0$; equivalent computational results were obtained independently by Kecik. ${ }^{13}$ Calculated and experimental ${ }^{14,15}$ reflectivity curves and colours for these compounds showed good agreement and the trends in colour as a function of the composition were well reproduced. In addition, the effect of disorder on the optical properties of $\mathrm{Au}_{0.5} \mathrm{Cu}_{0.5}$ was studied by comparing the dielectric function of the random solid solution, simulated using the supercell approach, with that of the ordered intermetallic compound, ${ }^{16}$ and the main spectral differences between the two different types of compounds were captured by the simulations.

In this work, first we establish a general computational approach that can be used for the photorealistic simulation of metals, showing how the reflectivity and colour of metallic crystals can be estimated by means of first-principles techniques. We then demonstrate through a systematic study on elemental metals and extensive comparisons with experimental data that the theoretical and numerical approximations adopted are able to reproduce the correct behaviour of the reflectivity curves and to capture the main differences in optical properties across the periodic table. Finally, we perform a similar study on metal alloys by considering different types of compounds, that is, ordered intermetallics, disordered solid solutions and heterogeneous alloys. In particular, we show through a comparison with experimental results that,

\footnotetext{
${ }^{1}$ Theory and Simulation of Materials (THEOS) and National Centre for Computational Design and Discovery of Novel Materials (MARVEL), École Polytechnique Fédérale de Lausanne, 1015 Lausanne, Switzerland. ${ }^{2}$ Institute of Condensed Matter and Nanosciences (IMCN), Université catholique de Louivain, 1348 Louvain-la-Neuve, Belgium. *email: nicola.marzari@epfl.ch
} 


\section{ColourWorkflow}

\section{Crystal structure}

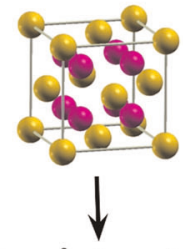

(i) Band structure

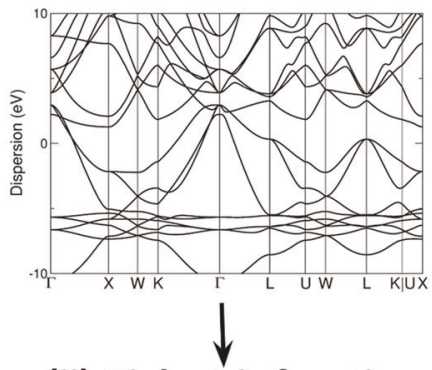

(ii) Dielectric function (interband + intraband)

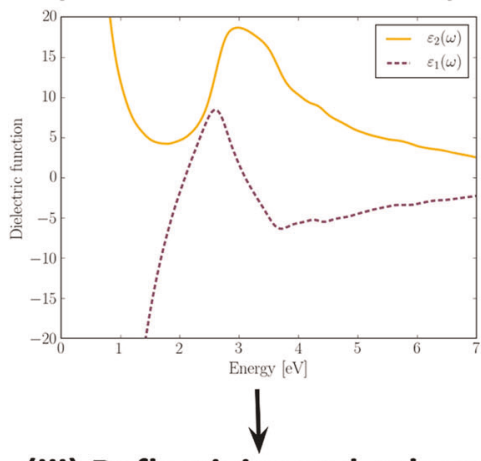

(iii) Reflectivity and colour
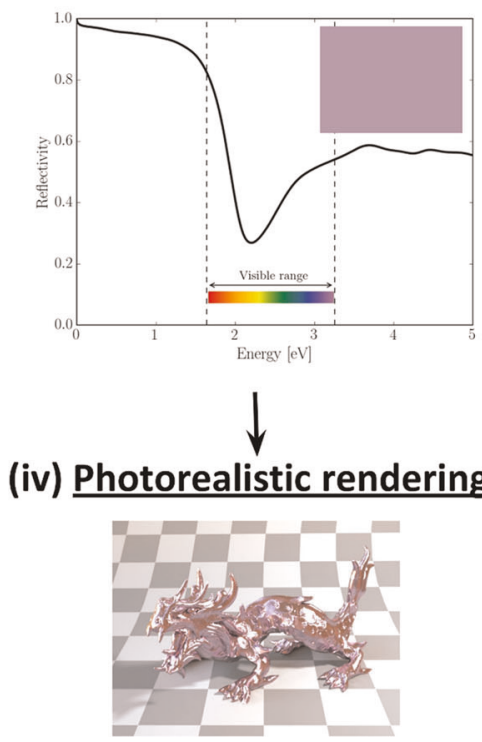

Fig. 1 Schematic representation of the workflow, named ColourWorkflow, designed to simulate the reflectivity and colour of a metallic material giving as input its crystal structure. if the appropriate methods are used for the simulation of the different types of compounds, (i) the simulated colours of known coloured intermetallics are in qualitative and most often in quantitative agreement with experiments and that (ii) one can reproduce the main colour trends in noble-metal-based binary alloys.

\section{RESULTS}

Computational approach

The computational workflow that allows one to obtain the reflectivity and colour of a given metal from an initial crystal structure, schematically depicted in Fig. 1, can be divided into four main computational steps: (i) evaluation of the electronic structure, (ii) calculation of the dielectric function, (iii) calculation of the reflectivity and colour and (iv) photorealistic rendering of the material.

The quantity we consider for the first-principles simulation of optical properties is the complex, wavevector- and frequencydependent, dielectric function $\varepsilon(\mathbf{q}, \omega)=\varepsilon_{1}(\mathbf{q}, \omega)+i \varepsilon_{2}(\mathbf{q}, \omega)$. In fact, the knowledge of the dielectric function gives then access to all the optical constants measurable by optical experiments, such as absorption coefficient and reflectivity.

Throughout this work the electronic structure is computed using density-functional theory (DFT) ${ }^{17}$ relying on the semi-local Perdew-Burke-Ernzerhof (PBE) exchange-correlation functional. ${ }^{18}$ We emphasize here that the electronic structure could alternatively be obtained with more accurate techniques; for example, the accuracy of the band structures could be improved by computing quasi-particle corrections on top of PBE results (typically at the GW level ${ }^{19-21}$ ), albeit at a largely increased computational cost. Another similar approach consists in using more complex exchange-correlation functionals to correct the intrinsic errors of the PBE band structures, with the so-called orbital-dependent functionals, such as the GLLBSC, ${ }^{22}$ SCAN $^{23}$ and Koopmans' complaint ${ }^{24}$ functionals, giving the most promising results (see, for instance, refs ${ }^{25-27}$ ). So, while in the present work we just rely on the Kohn-Sham (KS) PBE bands, ${ }^{28}$ the use of conceptually and quantitatively correct GW bands (or, similarly, bands computed with more complex exchange-correlation functionals) would take place seamlessly inside this workflow.

Subsequently, we calculate the dielectric function within the independent particle approximation (IPA), which amounts to neglecting (i) effects related to electron-hole interactions (excitonic effects), since these are effectively screened by the conduction electrons and (ii) effects related to the rapidly varying microscopic electric fields inside the material (local-field effects). The latter are rather small in highly homogeneous systems such as bulk simple metals. In alloys, in contrast, they can in principle be more important, although they only affect the intensity of the optical spectra (sometimes even by several tens of percent) but not their shape. So, they should have a limited effect on the actual simulation of colours. More precisely, given that local-field effects increase with the ionicity of the compounds, we expect that these might play a role in some particular intermetallic compounds (see, for instance, Blaber et al. ${ }^{29}$ for the case of alkali-noble intermetallics) but not in solid solutions (which, in general, have small ionic character).

In the optical regime, the momentum $\mathbf{q}$ transferred by the photon is negligible so that we can consider the optical limit, $\mathbf{q} \rightarrow \mathbf{0}$, of the expression for the IPA dielectric function $\varepsilon(\mathbf{q}, \omega)$. In general, the dielectric function still depends on the direction $\hat{\mathbf{q}}=$ $\mathbf{q} /|\mathbf{q}|$ of the perturbing electric field, and only for crystals with cubic symmetry, it is the same in every direction. In the optical limit it is convenient to divide the evaluation of the IPA dielectric function of metals into two separate contributions, an intraband Drude-like term $\varepsilon^{\text {intra }}(\hat{\mathbf{q}}, \omega)$ due to the conduction electrons at the 
Fermi surface and an interband term $\varepsilon^{\text {inter }}(\hat{\mathbf{q}}, \omega)$ due to vertical transitions between occupied and unoccupied bands, so that $\varepsilon(\hat{\mathbf{q}}, \omega)=\varepsilon^{\text {inter }}(\hat{\mathbf{q}}, \omega)+\varepsilon^{\text {intra }}(\hat{\mathbf{q}}, \omega)$. Using the solutions of the oneparticle Schrödinger equation for periodic systems, $H^{\mathrm{KS}}\left|\psi_{n \mathbf{k}}\right\rangle=$ $E_{n \mathbf{k}}\left|\psi_{n \mathbf{k}}\right\rangle$ (where $H^{\mathrm{KS}}$ is the KS Hamiltonian from DFT), the explicit expression of the IPA dielectric function can be written as ${ }^{30-32}$

$$
\begin{aligned}
\varepsilon^{\text {inter }}(\hat{\mathbf{q}}, \omega)= & 1-\frac{4 \pi}{V} \sum_{\mathbf{k}} \sum_{\substack{n, n^{\prime} \\
n \neq n^{\prime}}} \frac{\left|\left\langle\psi_{n^{\prime} \mathbf{k}}|\hat{\mathbf{q}} \cdot \mathbf{v}| \psi_{n \mathbf{k}}\right\rangle\right|^{2}}{\left(E_{n^{\prime} \mathbf{k}}-E_{n \mathbf{k}}\right)^{2}} \\
& \times \frac{f_{n \mathbf{k}}-f_{n^{\prime} \mathbf{k}}}{\omega-\left(E_{n^{\prime} \mathbf{k}}-E_{n \mathbf{k}}\right)+i \eta}, \\
\varepsilon^{\text {intra }}(\hat{\mathbf{q}}, \omega)= & -\frac{\omega_{D}^{2}(\hat{\mathbf{q}})}{\omega(\omega+i \gamma)},
\end{aligned}
$$

where we have defined the IPA Drude plasma frequency as

$$
\omega_{D}^{2}(\hat{\mathbf{q}})=\frac{4 \pi}{V} \sum_{\mathbf{k}} \sum_{n}\left|\left\langle\psi_{n \mathbf{k}}|\hat{\mathbf{q}} \cdot \mathbf{v}| \psi_{n \mathbf{k}}\right\rangle\right|^{2}\left(-\frac{\partial f_{n \mathbf{k}}}{\partial E_{n \mathbf{k}}}\right) .
$$

In the expressions above, $\mathbf{v}=-i\left[\mathbf{r}, H^{\mathrm{KS}}\right]$ is the velocity operator, $f_{n \mathbf{k}}$ is the Fermi-Dirac occupation function of the KS Bloch state $\left|\psi_{n \mathbf{k}}\right\rangle$ identified by band index $n$ and wavevector $\mathbf{k}$ within the Brillouin zone (BZ) and $V$ is the volume of the crystal. Instead, $\eta$ is an infinitesimal broadening introduced to perform the adiabatic switch of the perturbation within linear-response theory and, in practical calculations, it is used as an empirical broadening, which accounts for scattering processes, always present in real materials, and/or for finite experimental resolution. Similarly, $\gamma$ is an empirical broadening representing dissipation effects of the conduction electrons (see the Methods section for more details on the parameters effectively used in the simulations). A more extensive discussion on the first-principles theory of optical properties and the derivation of the expression of the IPA dielectric function in the optical limit (Eqs. (1), (2) and (3)) can be found in ref. ${ }^{33}$

As the most typical experimental situation is to have polycrystalline materials in which grains have random orientations, in the following we always deal with the dielectric function averaged over the three Cartesian directions

$\varepsilon(\omega)=\frac{\varepsilon(\hat{\mathbf{x}}, \omega)+\varepsilon(\hat{\mathbf{y}}, \omega)+\varepsilon(\hat{\mathbf{z}}, \omega)}{3}$,

so that we can drop the dependence on the direction $\hat{\mathbf{q}}$. Similarly, we also define a corresponding average IPA Drude plasma frequency as $\omega_{\mathrm{D}}^{2}=\left[\omega_{\mathrm{D}}^{2}(\hat{\mathbf{x}})+\omega_{\mathrm{D}}^{2}(\hat{\mathbf{y}})+\omega_{\mathrm{D}}^{2}(\hat{\mathbf{z}})\right] / 3$.

In order to compute the reflectivity from the knowledge of the dielectric function, we first introduce the refractive index $n(\omega)$ and the extinction coefficient $k(\omega)$ that are defined from the equation $[n(\omega)+i k(\omega)]^{2}=\varepsilon(\omega)$. The reflectivity at normal incidence and assuming a vacuum-material interface is then simply linked to $n(\omega)$ and $k(\omega)$ through the Fresnel equations of classical electromagnetism (see for example ref. ${ }^{34}$ ):

$R(\omega)=\frac{[n(\omega)-1]^{2}+k(\omega)^{2}}{[n(\omega)+1]^{2}+k(\omega)^{2}}$.

Eventually, we relate the reflectivity of a material to its perceived colour using the standard colour spaces introduced by the Commission Internationale de l'Eclairage (CIE) (http://www. cie.co.at/) for quantitative measures of colour. For this purpose, trichromatic theory gives the rigorous mathematical framework that permits to estimate the colour of an opaque material (e.g. a metal) by knowing its reflectivity $R(\lambda)$ for all the wavelengths $\lambda$ in the visible range (i.e. in the range $[380,780] \mathrm{nm}$ ), and to condense this information into three numbers, that is, the colour coordinates. ${ }^{35}$ In particular, according to the CIE 1931 standard colorimetric observer, the tristimulus values $(X, Y, Z)$, which define the $\mathrm{CIE}-X Y Z$ colour space, completely describe a colour stimulus and are given by the following integrals over the visible range:

$$
\begin{aligned}
& X=k \int_{380 \mathrm{~nm}}^{780 \mathrm{~nm}} \mathrm{~d} \lambda \bar{x}(\lambda) R(\lambda) S(\lambda), \\
& Y=k \int_{380 \mathrm{~nm}}^{780 \mathrm{~nm}} \mathrm{~d} \lambda \bar{y}(\lambda) R(\lambda) S(\lambda), \\
& Z=k \int_{380 \mathrm{~nm}}^{780 \mathrm{~nm}} \mathrm{~d} \lambda \bar{z}(\lambda) R(\lambda) S(\lambda),
\end{aligned}
$$

where $\bar{x}(\lambda), \bar{y}(\lambda)$ and $\bar{z}(\lambda)$ are the three so-called colour-matching functions and describe the chromatic response of the observer, being related to the sensitivity of the three different coloursensitive photoreceptors present in the human eye. $S(\lambda)$ is instead the spectral power distribution of one of the standard CIE illuminant (throughout this work the D65 illuminant is used which corresponds to average daylight), while the constant $k$ is chosen so that $Y=100$ for objects for which $R(\lambda)=1$ for all visible wavelengths.

In practice, it is more convenient to work within the CIELAB colour space rather than in the CIE-XYZ colour space, which is defined by three coordinates $\left(L^{*}, a^{*}, b^{*}\right)$ that are easily computed from the knowledge of the tristimulus values $(X, Y, Z)$ through a coordinate transformation. ${ }^{35}$ Indeed, since the CIELAB colour space is nearly uniform, euclidean distances can be used to approximately represent the perceived magnitude of colour differences between two objects in the same external conditions. Therefore, if $\left(L_{1}^{*}, a_{1}^{*}, b_{1}^{*}\right)$ and $\left(L_{2}^{*}, a_{2}^{*}, b_{2}^{*}\right)$ are the CIELAB coordinates of two objects, their colour difference is simply given by

$$
\Delta E=\sqrt{\left(L_{1}^{*}-L_{2}^{*}\right)^{2}+\left(a_{1}^{*}-a_{2}^{*}\right)+\left(b_{1}^{*}-b_{2}^{*}\right)^{2}} .
$$

Typically, a difference $\Delta E>1-2$ can be perceived by the human eye. In addition, we use photorealistic rendering, which is based on the solution of the light-transport equation, ${ }^{36}$ to simulate the actual appearance of an object made of a material with specified optical constants in the visible range within a realistic three-dimensional (3D) scene.

Our goal is to apply the computational approach described above to study metals in their crystalline form. From the point of view of first-principles calculations, elemental crystals are the easiest and most computationally efficient systems to simulate since they are periodic and their primitive cell, which typically consists of only a few atoms, can simply be taken as the simulation cell. For multi-component systems instead (in this work we focus on binary alloys), we distinguish different types of compounds according to their atomic configuration and microstructure. In particular, we consider the following three limiting cases: (i) perfectly ordered phases, that is, pure intermetallic compounds, (ii) perfectly disordered phases, that is, pure solid solutions and (iii) heterogeneous alloys, that is, alloys consisting of a mixture of two different phases. In the present study, we only deal with the colour of intrinsic bulk of metals. Defects (e.g. substitutional defects, vacancies, dislocations, etc.) or any type of surface effect (such as surface segregation) will obviously affect the colour of the material, but taking these effects into account is beyond the scope of this work. Indeed, in principle, the optical properties of a material with defects could be obtained by performing supercell simulations employing simulation cells of the appropriate size. Similarly, the optical properties of a material presenting surface segregation could be obtained by performing an additional simulation of a fictitious bulk presenting the modified composition of the surface, and then combining the optical properties of the two compositions by using a suitable surface model. However, such simulations are significantly more computationally expensive with respect to the case of pristine bulk systems and will thus be left for subsequent work. 
Table 1. Different types of compounds and corresponding simulation method used in this work for the first-principles simulation of these systems.

\begin{tabular}{ll}
\hline Binary alloys & Computational method \\
\hline Intermetallic compounds & Primitive cell \\
Solid solutions & Supercell (SQS) \\
Heterogeneous alloys & Bruggeman model \\
\hline
\end{tabular}

We use different simulation methods in order to properly model the reflectivity and colour of the three different types of compounds considered here, as summarized in Table 1. As for the case of elemental crystals, pure intermetallic compounds are periodic systems and are simply simulated in their primitive cell. On the other hand, we use the supercell approach, based on the use of special quasi-random structures $(\mathrm{SQS}),{ }^{37,38}$ to take into account effects related to disorder in the simulation of the optical properties of solid solutions (see Supplementary Discussion 1 for a comparison between the SQS supercell approach and the virtualcrystal approximation). Instead, for heterogeneous alloys made of two phases $a$ and $\beta$, we use the Bruggeman mode ${ }^{39}$ to estimate the optical properties of the alloy. Within the Bruggeman model, the dielectric function of the mixture, which we indicate as $\varepsilon_{\mathrm{Br}}(\omega)$, is given by the following expression:

$$
\left(1-x_{\beta}\right) \frac{\varepsilon_{\alpha}(\omega)-\varepsilon_{\mathrm{Br}}(\omega)}{\varepsilon_{\alpha}(\omega)+2 \varepsilon_{\mathrm{Br}}(\omega)}+x_{\beta} \frac{\varepsilon_{\beta}(\omega)-\varepsilon_{\mathrm{Br}}(\omega)}{\varepsilon_{\beta}(\omega)+2 \varepsilon_{\mathrm{Br}}(\omega)}=0,
$$

in terms of the dielectric functions $\varepsilon_{a}(\omega)$ and $\varepsilon_{\beta}(\omega)$ of the single phases, and where $x_{a}$ and $x_{\beta}$ (with $x_{a}+x_{\beta}=1$ ) are the fractions of the two phases present in the material. The dielectric function of the single phases can be obtained with the methods of Table 1 for intermetallic compounds and solid solutions.

In the following, we apply and validate the computational approach described here, and discuss its limitations, on several elemental metals and binary compounds.

\section{Elemental metals}

Figure 2 shows the comparison between IPA results and experimental data for the reflectivity curves of 18 elemental metals, focusing on frequencies centred around the visible range (i.e. in the range $[1.59,3.26] \mathrm{eV}$ ). Experimentally, we observe high and flat reflectivities along the visible spectrum for the "precious" transition metals (i.e. Rh, Ir and Pd), while we observe flat but slightly lower reflectivities for the other transition metals considered (i.e. V, Nb, Ta, Cr, Mo and W). As a consequence, in terms of CIELAB colour coordinates, metals in the first group have a large $C I E L A B$ brightness $L^{*}$ and thus whitish colour, while the others have smaller brightness and thus a more greyish colour (e.g. rhodium has $L^{*}=90$, while vanadium has $L^{*}=78$ ). An interesting exception among the transition metals is osmium, which shows a reflectivity curve that is low in the low-energy part of the visible spectrum, but then suddenly rises in the blue-violet part, thus giving a bluish tint to pure osmium. A similar behaviour is found also in tantalum, but the rise of the reflectivity curve in the blue-violet region is significantly smaller and, consequently, also the bluish tint of the material is less pronounced. Instead, the simple $s p$ metals lithium, potassium and aluminium all have very high and nearly flat reflectivity curves in the visible range (and therefore whitish colour), while in beryllium the intensity of the reflectivity is lower, and comparable to that of the transition metals (and thus having a greyish colour). Interestingly, the reflectivity curve of caesium decreases significantly within the visible range, so that red and yellow radiation is strongly reflected, while all other visible frequencies are absorbed, giving a yellow tint to the material. As clearly shown in Fig. 2, the IPA simulations reproduce these different features of the elemental metals. In contrast, for noble metals, while the characteristic drop in the reflectivity curve in the visible range (for $\mathrm{Cu}$ and $\mathrm{Au}$ ) or in the ultraviolet (for $\mathrm{Ag}$ ) is also reproduced by the simulations, it happens at smaller energies compared to experiments due to the well-known underestimation of the interband gap between valence $d$ bands and conduction sp bands of PBE band structures. This discrepancy can be corrected using approaches beyond DFT, such as the GW approximation of many-body perturbation theory. By correcting the DFT band energies at the $G_{0} W_{0}$ level, a quantitative agreement with respect to experiments is obtained for the optical spectra of $\mathrm{Cu}^{40}$ and $\mathrm{Ag}^{41}$ but not for $\mathrm{Au}$, for which $G_{0} W_{0}$ gives very similar results to PBE. ${ }^{42}$ For this latter case, the quasi-particle self-consistent GW (QSGW) ${ }^{43,44}$ approach is required for the occupied $5 d$ bands of gold to be lowered in energy by the right amount ${ }^{42}$ (to note also that, opposite to the PBE results, the use of the hybrid $\mathrm{HSE}^{45}$ exchange-correlation functional for gold opens the interband gap too much compared to experiments ${ }^{42}$ ).

A quantitative measure of the accuracy of the simulations can be obtained through the colour difference $\Delta E$ (given in Eq. (9)) with respect to experiments. Its average value is found to be $\langle\Delta E\rangle=6.4$. For a more qualitative visual comparison, Fig. 3 shows the simulated rendering of a metallic surface of elemental gold, osmium and caesium together with the appearance of experimental samples of the same materials. In gold, the shift of the reflectivity edge in the simulations with respect to experiments makes the rendered colour more reddish than the true red-yellow colour of pure gold. On the other hand, the bluish colour of osmium and the yellow colour of caesium are well reproduced by the IPA simulations.

Moreover, as shown in Table 2, the IPA results for the Drude plasma frequency are in good agreement both with experiments and with previous simulations ${ }^{32}$ performed at the same level of theory for some elemental metals.

From all these results, we conclude that the IPA approach applied on top of PBE band structures predicts the reflectivity and colour of elemental metals surprisingly well. Although the colour is not always in quantitative agreement with experiments, the shape and the main features of the experimental reflectivity curves are reproduced in elemental metals. These results are somewhat surprising because it is known that quasi-particle corrections modify significantly the PBE band structure in metals and the corrections are $k$-dependent ${ }^{40,41}$ (i.e. they do not act as a simple scissor operator). Nonetheless, these approximated simulations manage to capture the correct features of the optical constants. This can intuitively be understood by the fact that the dielectric function is given by the sum of all possible vertical transitions over all the BZ and small differences in the positions and features of the bands (like gradient and curvature) are averaged out in the spectra. In the special case of noble metals, the position of the occupied $d$ bands in PBE is not correct and, since there are no other allowed interband transitions in that energy range, the onset of interband optical absorption (i.e. $\left.\varepsilon_{2}^{\text {inter }}(\omega)\right)$ in PBE is also not at the correct position (similar to the case of semiconductors for which the PBE band gap is systematically underestimated $\left.{ }^{20}\right)$. On the other hand, the shape of $\varepsilon(\omega)$ for noble metals is reasonably well reproduced.

\section{Alloys}

In order to validate the theoretical approach used on binary compounds, we first compare the reflectivity and colour between simulations and experiments for known coloured intermetallic compounds, as previously done for elemental metals. Second, we check the predictive accuracy of the simulations by studying in noble-metal-based alloys both the trends in reflectivity with respect to composition (in $\mathrm{Ag}-\mathrm{Au}$ and $\mathrm{Ag}-\mathrm{Cu}$ ) and the differences 

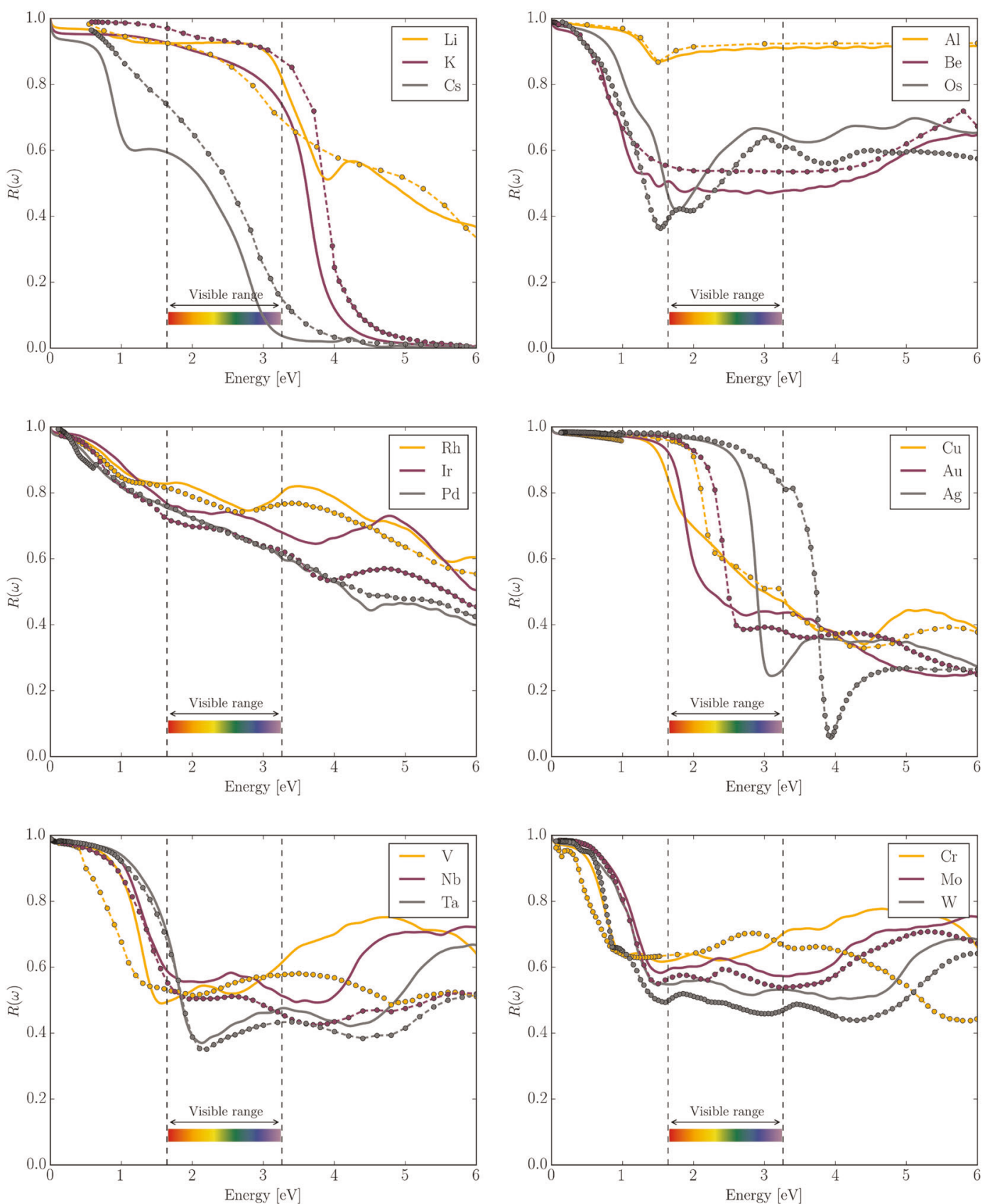

Fig. 2 Simulated (solid lines) and experimental (dot-dashed lines) reflectivities for $\mathbf{1 8}$ elemental metals. Experimental data are taken from ref. ${ }^{65}$ The two vertical dashed lines show the limits of the visible range.

in optical properties among different types of compounds for a given alloy composition (in $\mathrm{Au}-\mathrm{Cu}$ and $\mathrm{Ag}-\mathrm{Cu}$ ).

Intermetallics. We first simulate the reflectivity and colour of intermetallic compounds that are experimentally known to be coloured. The compounds studied are the purple $\mathrm{AuAl}_{2}$, blue Auln $n_{2}$, bluish $\mathrm{AuGa}_{2}$, yellow $\mathrm{PtAl}_{2}$, red Pdln, blue-grey $\mathrm{NiSi}_{2}$ and dark blue $\mathrm{CoSi}_{2}{ }^{6,46}$ All these intermetallics have cubic symmetry: $\mathrm{AuAl}_{2}, \mathrm{AuGa}_{2}, \mathrm{Auln}_{2}, \mathrm{PtAl}_{2}, \mathrm{CoSi}_{2}$ and $\mathrm{NiSi}_{2}$ crystallize in the facecentred cubic (FCC) $\mathrm{CaF}_{2}$ prototype structure (space group $\mathrm{Fm} \overline{\mathrm{3}} \mathrm{m}$ ), while Pdln crystallizes in the BCC CsCl prototype structure (space group $P m \overline{3} m)$.

As shown in Fig. 4, the experimental shape of the reflectivity curve for the coloured intermetallics is well reproduced by the simulations. The colour differences between simulations and experiments are summarized in Table 3, where the comparison with other first-principles simulations ${ }^{11,12}$ is also reported. The agreement with previous simulations is satisfactory and, moreover, we reproduce the true colour of the intermetallic compounds studied (although the CIELAB brightness is typically overestimated by the simulations). For example, the comparison between photorealistic rendering and real material samples clearly shows that the simulations predict the correct colours of purple $\mathrm{AuAl}_{2}$, bluish $\mathrm{AuGa}_{2}$ and yellow $\mathrm{PtAl}_{2}$ (see Fig. 5). The characteristic colours of these highly symmetric intermetallic compounds are due to selective optical absorption in confined regions of the visible spectrum. ${ }^{46}$ For the gold compounds, the optical absorption inside the visible range is given by transitions from $s p$ conduction states below the Fermi level to unoccupied states above the Fermi level. The bands originating from the $5 d$ states of 

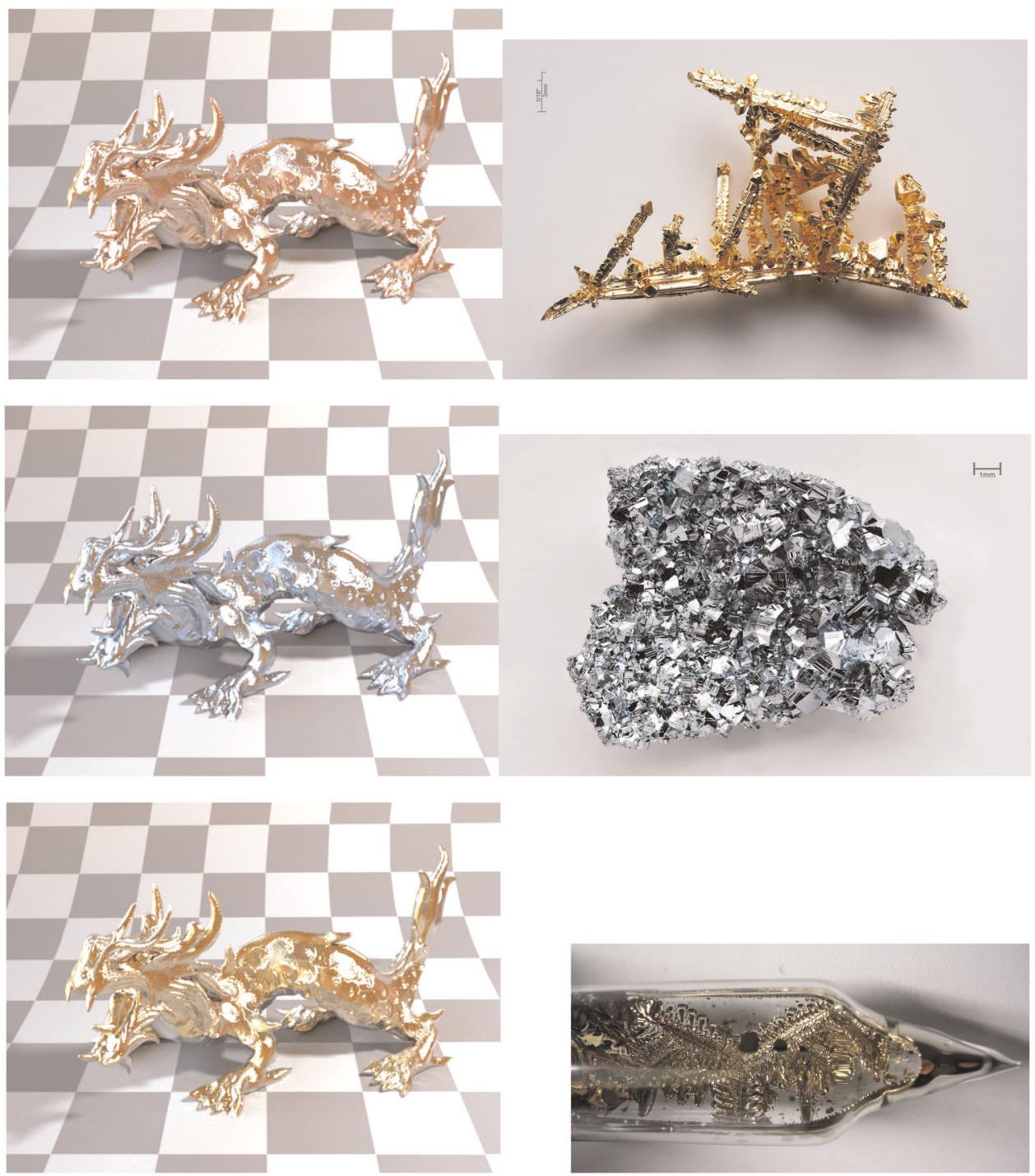

Fig. 3 Comparison between the simulated rendering of a metallic surface (left panel) and real samples (right panel) of pure gold (top), osmium (centre) and caesium (bottom). Photos of the gold and osmium samples are reproduced with the courtesy of Heinrich Pniok (www. pse-mendelejew.de) and are published under the Free Art License (http://artlibre.org/licence/lal/en/). Photo of the caesium sample is from the Dennis "S.K." collection and is published under the GNU Free Documentation License (https://www.gnu.org/licenses/fdl.html).

gold, that are problematic in the study of elemental gold, are located at $\sim 5 \mathrm{eV}$ below the Fermi level and these do not contribute to the characteristic colours of these compounds. ${ }^{47}$ This explains the better agreement with experiments found for the gold intermetallics compounds compared to the case of elemental gold.

$A u-A g-C u$. The Au-Ag-Cu system is an ideal test case for the application of the computational approach described above to alloys since (i) several experimental optical data on this system are available, (ii) its constituent binaries show very different behaviours in terms of phase stability and so different types of compounds are observed and (iii) it is the basis of the most common jewellery and dental alloys in use today. Concerning the phase stability of the constituent binaries, Ag is completely soluble in $\mathrm{Au}$, thus $\mathrm{Au}$ and $\mathrm{Ag}$ form solid solutions for each composition and no long-range order is observed at low temperatures. Also Au and $\mathrm{Cu}$ form solid solutions over all concentrations at high temperatures, but, for certain composition ranges, ordered intermetallic phases can be obtained at lower temperatures. In particular, the known intermetallic compounds are the cubic
$\mathrm{AuCu}_{3}$ and $\mathrm{Au}_{3} \mathrm{Cu}$ (space group $\mathrm{Pm} \overline{3} m$ ), the low-temperature phase $\mathrm{AuCu}(\mathrm{I})$ (space group $\mathrm{P} 4 / \mathrm{mmm}$ ) and the high-temperature phase $\mathrm{AuCu}$ (II) (space group Imma). The phase diagram of $\mathrm{Ag}-\mathrm{Cu}$ instead exhibits eutectic behaviour with a wide miscibility gap and the system tends to segregate in phases of nearly pure $\mathrm{Ag}$ and pure $\mathrm{Cu}$ at room temperature. ${ }^{48}$

We study the effect of composition on the reflectivity of the AgAu system and compare experimental data of solid solutions with SQS simulations. Figure 6 shows that the gradual shift to lower wavelengths of the reflectivity edge of gold by increasing the Ag content is reproduced by the simulations. However, as already discussed above for the case of elemental noble metals, the position of the reflectivity edge in IPA simulations based on PBE band structures does not correspond to the experimental one, but it is instead systematically shifted to longer wavelengths for each atomic concentration $x$ considered. Although the simulations are not in quantitative agreement with experiments, the qualitative trends in reflectivity, and thus in colour, with respect to the alloy composition of $\mathrm{Ag}$-Au are reproduced. Similarly, we simulate the optical properties of $\mathrm{Ag}_{1-x} \mathrm{Cu}_{x}$ two-phase alloys by employing the Bruggeman model described above and study also in this system 
the effect of composition on the reflectivity. The $a$ and $\beta$ phases entering in the expression for the alloy dielectric function $\varepsilon_{\mathrm{Br}}(\omega)$ of Eq. (10) are assumed to be elemental $\mathrm{Ag}$ and elemental $\mathrm{Cu}$, respectively. And the dielectric functions of the two constituent elements are taken from the simulations of elemental metals discussed above. As shown in Fig. 7, Ag additions in Cu increase the reflectivity at wavelengths shorter than the reflectivity edge of elemental $\mathrm{Cu}$ but do not shift, as it happens in $\mathrm{Ag}-\mathrm{Au}$ solid solutions, the position of the edge. The Bruggeman model provides the correct trend with composition, but the effect on the drop in the reflectivity is less evident because the reflectivity edge of elemental $\mathrm{Cu}$ in IPA simulations is less steep than the experimental one. Note that the application of the Bruggeman model to experimental data of the dielectric function of elemental $\mathrm{Ag}$ and elemental $\mathrm{Cu}$ gives very good agreement with experimental data for the two-phase alloy and validates the use of the model.

Summarizing, for Ag-Au solid solutions, where there is a gradual shift of the reflectivity edge by varying alloying additions from elemental $\mathrm{Au}$ to elemental Ag, the colour of the alloy changes from red-yellow to yellow, pale greenish-yellow and eventually

Table 2. Computed values of the IPA Drude plasma frequency $\omega_{D}$ (in eV) compared to previous simulations ( $\mathrm{Harl}^{32}$ ) and experiments (Exp.).

\begin{tabular}{lccl}
\hline Element & This work & Harl $^{32}$ & Exp. \\
\hline $\mathrm{Cu}$ & 8.8 & 9.1 & $8.8,8.9$ \\
$\mathrm{Ag}$ & 8.9 & 9.2 & $8.9 \pm 0.2,{ }^{70} 8.9$ \\
$\mathrm{Au}$ & 8.6 & 9.0 & $8.45^{71} 8.7$ \\
$\mathrm{Li}$ & 6.4 & 6.5 & 6.4 \\
$\mathrm{Na}$ & 6.0 & 5.9 & 5.7 \\
$\mathrm{Ca}$ & 4.1 & 4.3 & 5.7 \\
$\mathrm{Al}$ & 12.5 & 12.6 & $12.3,12.5$ \\
$\mathrm{Rh}$ & 9.6 & 10.1 & \\
$\mathrm{Pd}$ & 7.0 & 7.4 & \\
$\mathrm{Pt}$ & 8.4 & 8.8 & \\
\hline
\end{tabular}

The experimental values with no explicit reference are extracted from the data reported in ref. ${ }^{32}$ For transition metals there are no experimental data available because, due to the presence of interband transitions even at vanishingly small frequencies, the Drude plasma frequency cannot be extracted by fitting experimental optical data to the Drude model, even at very low energies

white of pure Ag. Au-Cu solid solutions show a similar behaviour ${ }^{49}$ and the colour of the alloy changes from red-yellow to reddish and eventually red of pure $\mathrm{Cu}$. Instead, in $\mathrm{Ag}$-Cu two-phase alloys there is no shift of the reflectivity edge but, for all wavelengths in the visible range below the reflectivity edge of elemental $\mathrm{Cu}$, the reflectivity curve rises roughly uniformly so that the colour of Ag$\mathrm{Cu}$ changes from the red of pure $\mathrm{Cu}$ to reddish and then directly to whitish and white of pure Ag. ${ }^{6}$

After considering the effect of composition on the reflectivity of binary alloys, we now study the effect of different types of compounds for a given fixed atomic concentration $x$ directly on the dielectric function of the $\mathrm{Au}-\mathrm{Cu}$ and $\mathrm{Ag}$-Cu systems. Indeed, for $\mathrm{Au}-\mathrm{Cu}$ at the composition $x=0.81$, experimental data are available in the literature for the optical absorption of both the solid solution and the intermetallic compound $\mathrm{AuCu}_{3}{ }^{49}$ Analogously, for $\mathrm{Ag}-\mathrm{Cu}$ at the composition $x=0.30$, experimental data are available for both a segregated two-phase sample made of a pure $\mathrm{Cu}$ phase and a pure Ag phase, and for a metastable solid solution obtained by vapour quenching. ${ }^{49}$ We compare the optical absorption of the $\mathrm{Au}_{1-x} \mathrm{Cu}_{x}$ solid solution, at $x=0.81$ in experiments and at $x=0.75$ in simulations, with the optical absorption of the intermetallic compound appearing around the composition $x=0.75$, that is, the cubic $\mathrm{AuCu}_{3}$ phase. The purpose of this comparison is to study the differences in optical properties between ordered and disordered phases. As shown in Fig. 8, the optical absorption of the intermetallic compound is very similar to the one of the random alloy with the notable exception of the presence of an additional peak at around $3.6 \mathrm{eV}$, which is missing in $\varepsilon_{2}(\omega)$ for the solid solution. The comparison of the SQS results

Table 3. Colour differences in CIELAB space between simulated colours (present work) and experimental colours ${ }^{12,14,46,66}$ derived from reflectivity data, $\Delta E_{\exp }$, and between simulated colours (present work) and previously published simulations, ${ }^{11,12} \Delta E_{\text {sim }}$

\begin{tabular}{lll}
\hline Compound & $\Delta E_{\exp }$ & $\Delta E_{\text {sim }}$ \\
\hline $\mathrm{AuAl}_{2}$ & $11^{14}$ & $8^{12} 4^{11}$ \\
$\mathrm{AuGa}_{2}$ & $2^{14}$ & $1^{11}$ \\
$\mathrm{Auln}_{2}$ & $4^{14}$ & $1^{11}$ \\
$\mathrm{PtAl}_{2}$ & $12^{12}$ & $2^{12}$ \\
$\mathrm{COSi}_{2}$ & $3^{46}$ & \\
$\mathrm{NiSi}_{2}$ & $5^{66}$ & \\
$\mathrm{Pdln}$ & $10^{46}$ & \\
\hline
\end{tabular}
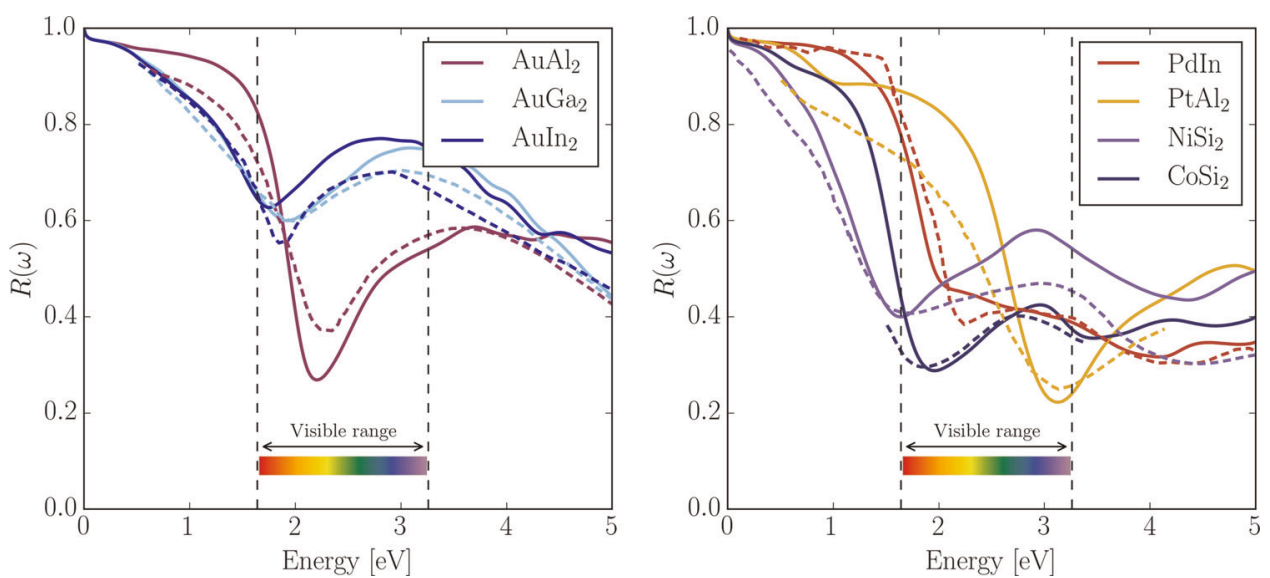

Fig. 4 Simulated (solid lines) and experimental (dashed lines) reflectivities of coloured intermetallics. Experimental data are taken from ref. ${ }^{14}$ for $\mathrm{AuAl}_{2}, \mathrm{AuGa}_{2}$ and $\mathrm{Auln}_{2}$, from ref. ${ }^{12}$ for $\mathrm{PtAl}_{2}$, from ref. ${ }^{66}$ for $\mathrm{NiSi}_{2}$ and from ref. ${ }^{46}$ for $\mathrm{CoSi}{ }_{2}$ and Pdln. The two vertical dashed lines show the limits of the visible range. 

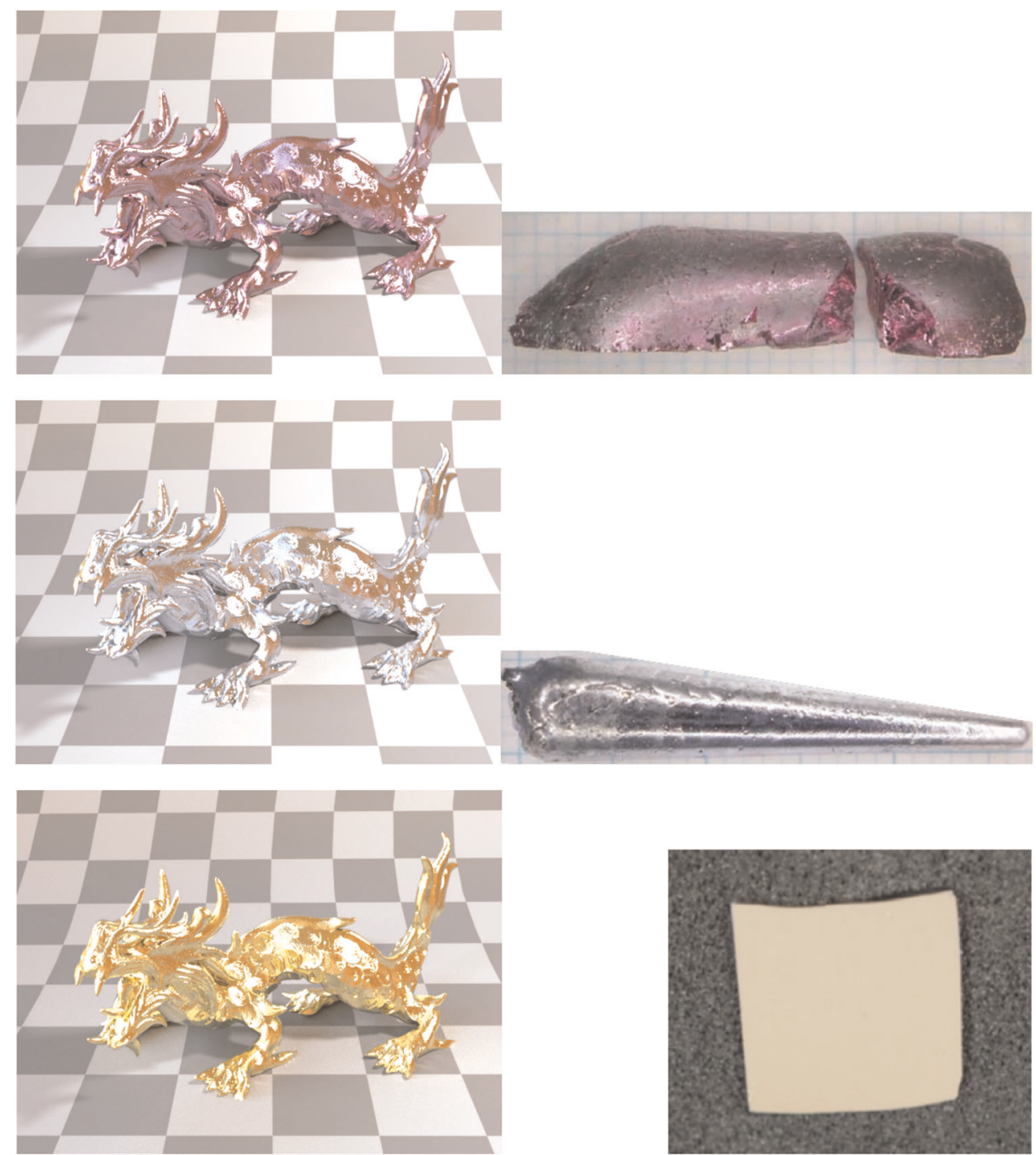

Fig. 5 Comparison between the simulated rendering of a metallic surface (left panel) and real samples (right panel) of the intermetallic compounds $\mathbf{A u A l}_{2}$ (top), $\mathbf{A u G a}_{2}$ (centre) and $\mathrm{PtAl}_{2}$ (bottom). Images of the $\mathrm{AuAl}_{2}$ and $\mathrm{AuGa}_{2}$ samples are adapted from ref., ${ }^{67}$ while image of the $\mathrm{PtAl}_{2}$ sample is adapted from ref.
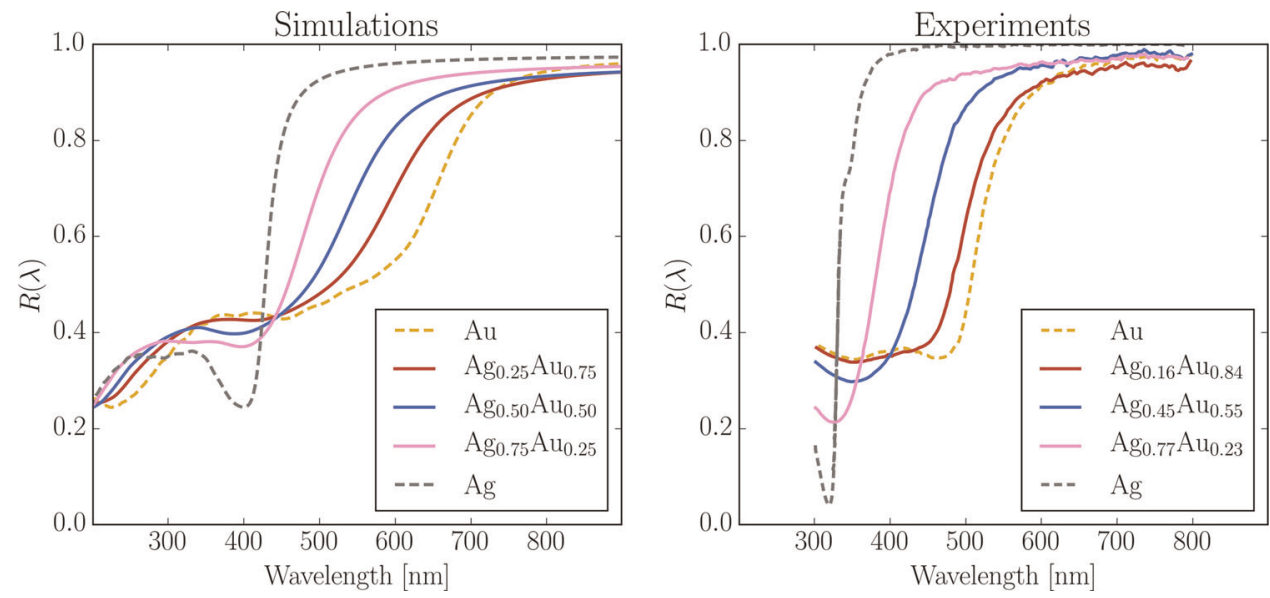

Fig. 6 Comparison of the trends in composition of the reflectivity inside the visible spectrum for Ag-Au solid solutions between SQS simulations (left panel) and experiments ${ }^{68}$ (right panel). For reference, we also report the reflectivity curves of elemental Au and elemental $\mathrm{Ag}$ (dashed lines). To note that the alloy compositions of experiments and simulations are not exactly the same. 

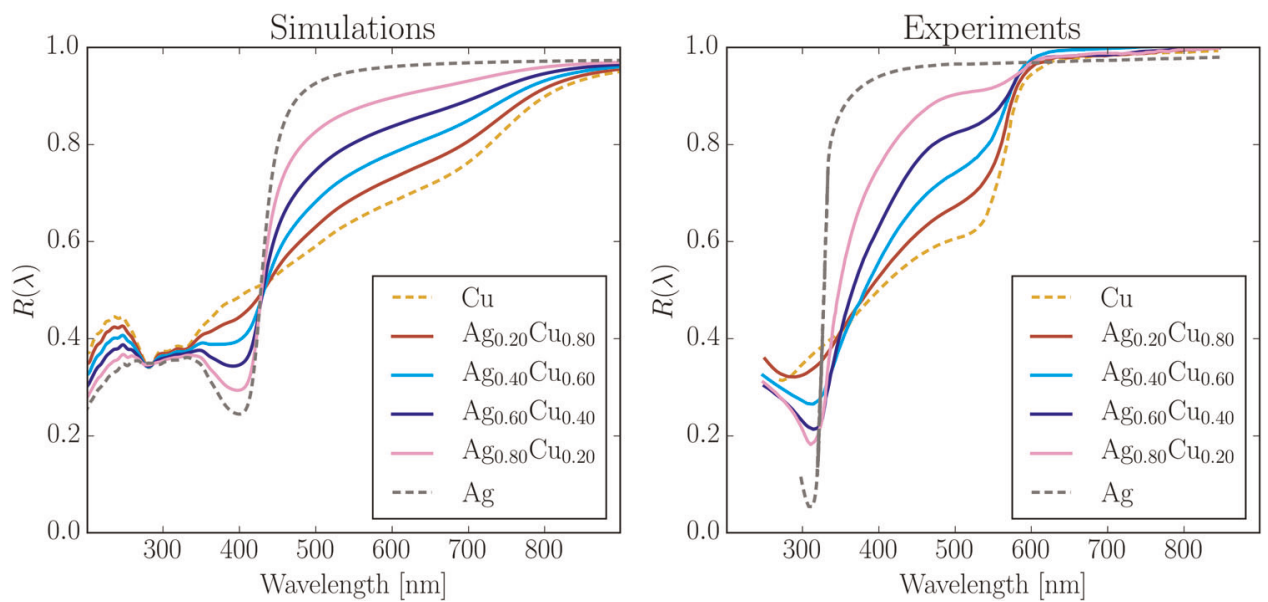

Fig. 7 Comparison of the trends in composition of the reflectivity inside the visible spectrum for Ag-Cu two-phase alloys between simulations (left panel) and experiments ${ }^{69}$ (right panel). The results of the simulations are obtained using the Bruggeman model in which the two phases of the system are assumed to be elemental Ag and elemental $\mathrm{Cu}$ (dashed lines).
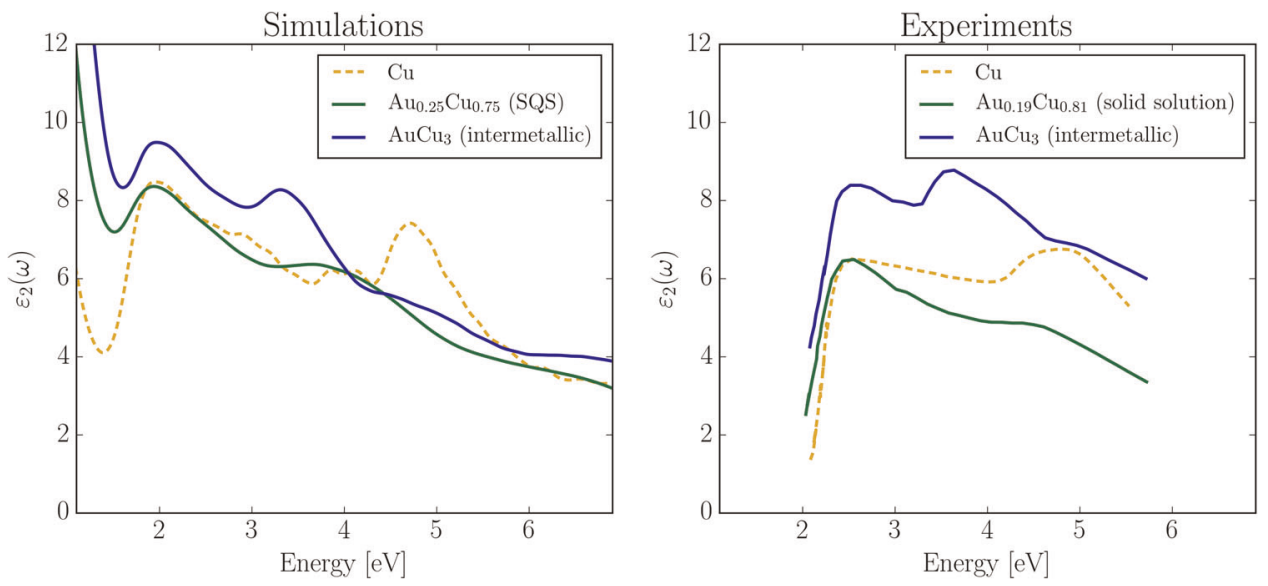

Fig. 8 Comparison of $\varepsilon_{2}(\omega)$ for $\mathrm{Au}_{1-x} \mathrm{Cu}_{x}$ between simulations (left panel) and experiments ${ }^{49}$ (right panel) for both the solid solution and the intermetallic phase $\mathrm{AuCu}_{3}$ (at $\mathbf{x}=\mathbf{0 . 7 5}$ in the simulations and at $\mathbf{x}=\mathbf{0 . 8 1}$ in the experiments). For reference, we also report $\varepsilon_{2}(\omega)$ of elemental $\mathrm{Cu}$ (dashed lines). Experimental and simulated curves have been arbitrarily shifted along the vertical axis for clarity in the comparison.

for the disordered alloy with the simulated results of the intermetallic compound shows that the simulations clearly capture this small difference. Nonetheless, we underline that there is no significant change in the resulting colour between ordered and disordered alloy for this system because the position of the onset of optical absorption is not modified by the presence of long-range order, and thus neither is the colour.

Similarly, Fig. 9 reports the comparison between the optical absorption of the $\mathrm{Ag}_{1-x} \mathrm{Cu}_{x}$ two-phase alloy, at $x=0.70$ in experiments and at $x=0.75$ in simulations, with respect to that of the metastable solid solution having the same composition. In the two-phase alloy, where the alloy optical properties are well approximated by a combination of those of pure $\mathrm{Cu}$ and pure $\mathrm{Ag}$ (Bruggeman model), we observe two onsets of absorption: the first one at $\sim 2.1 \mathrm{eV}$ corresponding to the absorption edge of pure $\mathrm{Cu}$ and the second one at $\sim 4.0 \mathrm{eV}$ corresponding to the absorption edge of pure Ag. The optical absorption of the solid solution instead is very similar to the one of pure $\mathrm{Ag}$, but, in addition, we observe the presence of a supplementary broad peak at energies below the onset of absorption of pure Ag due to $\mathrm{Cu}$ impurity states. The SQS results for the solid solution and the results of the Bruggeman model applied on the IPA dielectric function of elemental $\mathrm{Ag}$ and $\mathrm{Cu}$ reproduce the two different trends, although the SQS shows a small blueshift of the peak that follows the absorption edge of pure $\mathrm{Ag}$, which is not observed experimentally.

\section{DISCUSSION}

We have shown that the theoretical methods and approximations considered in this paper, that is, IPA optical spectra computed on top of the DFT-PBE electronic structure, can be employed in systematic studies on the optical properties of metals in order to predict trends in real metallic systems and to help the search for novel materials with specific optical properties, and therefore also colours, by exploring the composition space through the computational screening of materials. ${ }^{33}$ Moreover, this work could help stimulate future studies aiming to achieve the photorealistic simulation of different types of materials by means of firstprinciples techniques. For example, the systematic validation of the approach performed on elemental metals and binary alloys can be seen as a necessary preliminary step for the photorealistic simulation of more complex metallic alloys having a larger number of constituent elements, such as ternaries, quaternaries, and so on, which are more relevant for technological applications (e.g. superalloys and high-entropy alloys). 

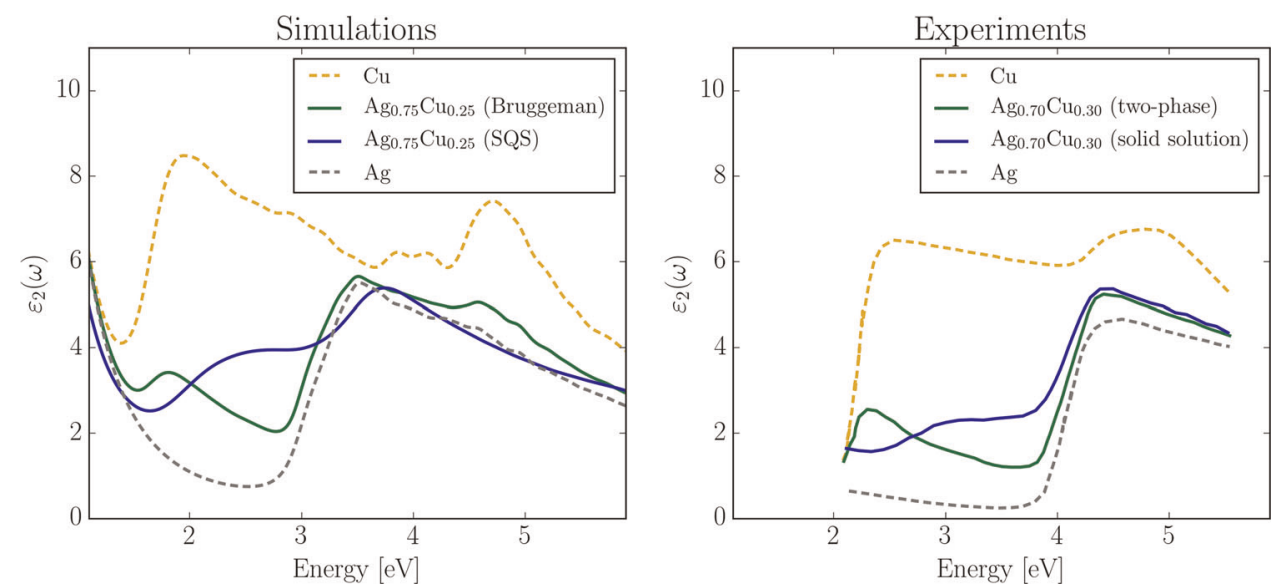

Fig. 9 Comparison of $\varepsilon_{2}(\omega)$ for $\mathrm{Ag}_{1-x} \mathrm{Cu}_{x}$ between simulations (left panel) and experiments ${ }^{49}$ (right panel) for both the solid solution and the two-phase alloy (at $x=0.25$ in the simulations and at $x=0.30$ in the experiments).

\section{METHODS}

\section{Workflow}

All DFT calculations are performed with the Quantum ESPRESSO distribution, ${ }^{50}$ which is based on the plane-wave pseudopotential method for the numerical solution of the KS equations. We use Shirley's interpolation method ${ }^{51,52}$ as implemented in the SIMPLE $\operatorname{code}^{53}$ to evaluate the IPA dielectric function of metals, including both interband and intraband contributions. Photorealistic rendering is performed with the Mitsuba renderer (http://www.mitsuba-renderer.org/). Pseudopotentials and plane-wave cutoffs are chosen according to the results of the standard solid-state pseudopotential (SSSP) protocol $^{54}$ in order to have reliable and converged band structures as the starting ingredients for the evaluation of the IPA dielectric function. Since the SIMPLE code supports only norm-conserving pseudopotentials, we use optimized normconserving Vanderbilt ${ }^{55}$ pseudopotentials from the $\mathrm{SG}^{5} 5^{56}$ and PseudoDojo $^{57}$ PBE pseudopotential libraries for all elements considered (see Supplementary Discussion 2 for more details on the choice of the pseudopotentials from the SSSP database of tests). For the purpose of automation, the sequence of calculations required by the computational approach described in this work is implemented as a workflow within the framework of the AiiDA ${ }^{58}$ infrastructure for computational science. Owing to this ColourWorkflow (see Fig. 1), it is possible, giving as input a generic crystal structure, to obtain directly as output the reflectivity and colour of a given material.

In all simulations, relativistic effects are accounted for at the scalarrelativistic level (see Supplementary Discussion 3 for an analysis on the effect of spin-orbit coupling on the optical properties of heavy elements), while the IPA dielectric function is always evaluated by including the nonlocal contribution of the pseudopotentials in the computation of the velocity matrix elements, as implemented in SIMPLE.

\section{Elemental metals}

All calculations on elemental metals are performed on the ground-state crystal structures at zero temperature, as provided in ref. ${ }^{59}$ The equilibrium volume of each structure corresponds to the reference PBE value obtained by extensively tested all-electron calculations for the equation of state. ${ }^{60}$ If needed, the crystal structures are reduced to the primitive cell using the spglib library (https://atztogo.github.io/spglib/). Spin polarization is not included in our calculations. In the selfconsistent DFT calculations for the evaluation of the ground-state density, we use a Monkhorst-Pack grid ${ }^{61}$ of $24 \times 24 \times 24$ and a cold smearing $^{62}$ of $0.02 \mathrm{Ry}$. In the non self-consistent band structure calculations needed for the construction of the Shirley's basis, we use a uniform $k$-grid of $2 \times 2 \times 2$ including the seven periodic images of the $\Gamma$-point of the BZ and at least 30 empty conduction bands. From a convergence study on the dielectric function, we decided to employ an interpolation $k$-grid of $64 \times 64 \times 64$ and $\eta=\gamma=0.1 \mathrm{eV}$ in SIMPLE for each elemental metal considered, with the exception of elemental aluminium for which, because of a very slow convergence of $\varepsilon^{\text {inter }}(\omega)$ with respect to $k$-point sampling, the interpolation $k$-grid used is
$80 \times 80 \times 80$ and $\eta$ is set to $0.2 \mathrm{eV}$. The Shirley's basis is constructed setting the threshold for the Gram-Schmidt orthonormalization algorithm equal to 0.0075 a.u. (input variable named $s_{b}$ in SIMPLE).

\section{Alloys}

For the simulation of all binary compounds considered, we always use as plane-wave cutoff the largest value between the plane-wave cutoffs of the two constituent elements, as taken from Supplementary Table 1. The Shirley's basis is constructed setting $s_{b}=0.01$ a.u. in SIMPLE and considering a number of empty bands at least equal to the number of occupied bands. We choose the interpolation k-grid to be used in the evaluation of the dielectric function in terms of a $k$-point density, which is defined as the maximum distance between adjacent $k$-points along the reciprocal axes (in $\AA^{-1}$ ). For all the seven cubic intermetallic compounds considered, we select as $k$-point density $0.04 \AA^{-1}$. With this choice, the number of $k$-points included in the uniform $k$-grids is of the order $O\left(10^{5}\right)$, which correspond to uniform $k$-grids in the range from $46 \times 46 \times 46$ up to $56 \times 56 \times 56$.

All the SQSs used in this work to simulate solid solutions of the systems $\mathrm{Ag}_{1-x} \mathrm{Au}_{x}, \mathrm{Au}_{1-x} \mathrm{Cu}_{x}$ and $\mathrm{Ag}_{1-x} \mathrm{Cu}_{x}$ are generated with the ATAT package. $^{63,64}$ Since we consider only the simple stoichiometric ratios $x=0.25,0.5,0.75$, we use small FCC SQSs with 16 atoms per cell. The interpolation $k$-grid is set according to a $k$-point density of $0.04 \AA^{-1}$ (corresponding roughly to 11,000 points in the BZ).

\section{DATA AVAILABILITY}

The data that support the findings of this study are available from the corresponding authors upon reasonable request.

\section{CODE AVAILABILITY}

The source code of the ColourWorkflow and the input scripts necessary in order to reproduce the simulations performed for this work are available at https://github. com/giprandini/colour-workflow.

Received: 7 June 2019; Accepted: 22 November 2019; Published online: 20 December 2019

\section{REFERENCES}

1. Bilokur, M., Gentle, A., Arnold, M. D., Cortie, M. B. \& Smith, G. B. High temperature spectrally selective solar absorbers using plasmonic AuAl2:AIN nanoparticle composites. Solar RRL 1, 1700092 (2017).

2. Guo, C., Sun, T., Cao, F., Liu, Q. \& Ren, Z. Metallic nanostructures for light trapping in energy-harvesting devices. Light Sci. Appl. 3, e161 (2014).

3. Yun, J. Ultrathin metal films for transparent electrodes of flexible optoelectronic devices. Adv. Funct. Mater. 27, 1606641 (2017). 
4. Ren, X., Li, X. \& Choy, W. C. Optically enhanced semi-transparent organic solar cells through hybrid metal/nanoparticle/dielectric nanostructure. Nano Energy 17, 187-195 (2015)

5. Hatwar, T. K., Tyan, Y. S. \& Brucker, C. F. High-performance Co/Pt multilayer magneto-optical disk using ultrathin seed layers. J. Appl. Phys. 81, 3839-3841 (1997).

6. Cretu, C. \& van der Lingen, E. Coloured gold alloys. Gold Bull. 32, 115-126 (1999).

7. Maksimov, E. G., Mazin, I. I., Rashkeev, S. N. \& Uspenski, Y. A. First-principles calculations of the optical properties of metals. J. Phys. F 18, 833 (1988).

8. Werner, W. S. M., Glantschnig, K. \& Ambrosch-Draxl, C. Optical constants and inelastic electron-scattering data for 17 elemental metals. J. Phys. Chem. Ref. Data 38, 1013-1092 (2009).

9. Blaber, M. G., Arnold, M. D. \& Ford, M. J. Optical properties of intermetallic compounds from first principles calculations: a search for the ideal plasmonic material. J. Phys. Condens. Matter 21, 144211 (2009).

10. Keast, V. J., Barnett, R. L. \& Cortie, M. B. First principles calculations of the optical and plasmonic response of $\mathrm{Au}$ alloys and intermetallic compounds. J. Phys. Condens. Matter 26, 305501 (2014).

11. Keast, V. J., Birt, K., Koch, C. T., Supansomboon, S. \& Cortie, M. B. The role of plasmons and interband transitions in the color of $\mathrm{AuAl}_{2}, \mathrm{Auln}_{2}$, and $\mathrm{AuGa}_{2}$. Appl. Phys. Lett. 99, 111908 (2011).

12. Keast, V., Zwan, B., Supansomboon, S., Cortie, M. \& Persson, P. O. A. AuAl 2 and $\mathrm{PtAl}_{2}$ as potential plasmonic materials. J. Alloy. Comp. 577, 581-586 (2013).

13. Kecik, D. First-Principles Investigation of the Optical Properties of Gold and its Alloys. Ph.D. thesis, EPF Lausanne (2013).

14. Vishnubhatla, S. S. \& Jan, J. P. Optical properties of the intermetallic compounds $\mathrm{AuAl}_{2}, \mathrm{AuGa}_{2}$ and Auln 2 . Philos. Mag. 16, 45-50 (1967).

15. Furrer, A. \& Spolenak, R. Colors of thin films of binary and ternary gold- and platinum-based alloys. Acta Mater. 66, 241-250 (2014).

16. De Silva, K. S. B., Gentle, A., Arnold, M., Keast, V. J. \& Cortie, M. B. Dielectric function and its predicted effect on localized plasmon resonances of equiatomic Au-Cu. J. Phys. D 48, 215304 (2015).

17. Hohenberg, P. \& Kohn, W. Inhomogeneous electron gas. Phys. Rev. 136, B864-B871 (1964).

18. Perdew, J., Burke, K. \& Ernzerhof, M. Generalized gradient approximation made simple. Phys. Rev. Lett. 77, 3865-3868 (1996).

19. Hybertsen, M. S. \& Louie, S. G. Electron correlation in semiconductors and insulators: band gaps and quasiparticle energies. Phys. Rev. B 34, 5390-5413 (1986).

20. Onida, G., Reining, L. \& Rubio, A. Electronic excitations: density-functional versus many-body Green's-function approaches. Rev. Mod. Phys. 74, 601-659 (2002).

21. Reining, L. The GW approximation: content, successes and limitations. Wiley Interdiscip. Rev. 8, e1344 (2017).

22. Kuisma, M., Ojanen, J., Enkovaara, J. \& Rantala, T. T. Kohn-Sham potential with discontinuity for band gap materials. Phys. Rev. B 82, 115106 (2010).

23. Sun, J., Ruzsinszky, A. \& Perdew, J. P. Strongly constrained and appropriately normed semilocal density functional. Phys. Rev. Lett. 115, 036402 (2015).

24. Dabo, l. et al. Koopmans' condition for density-functional theory. Phys. Rev. B 82, 115121 (2010)

25. Yan, J., Jacobsen, K. W. \& Thygesen, K. S. Conventional and acoustic surface plasmons on noble metal surfaces: a time-dependent density functional theory study. Phys. Rev. B 86, 241404 (2012).

26. Yang, Z.-h, Peng, H., Sun, J. \& Perdew, J. P. More realistic band gaps from metageneralized gradient approximations: only in a generalized Kohn-Sham scheme. Phys. Rev. B 93, 205205 (2016).

27. Nguyen, N. L., Colonna, N., Ferretti, A. \& Marzari, N. Koopmans-compliant spectral functionals for extended systems. Phys. Rev. X 8, 021051 (2018).

28. Kohn, W. \& Sham, L. J. Self-consistent equations including exchange and correlation effects. Phys. Rev. 140, A1133-A1138 (1965).

29. Blaber, M. G., Arnold, M. D. \& Ford, M. J. Designing materials for plasmonic systems: the alkali-noble intermetallics. J. Phys. Condens. Matter 22, 095501 (2010).

30. Marini, A., Onida, G. \& Del Sole, R. Plane-wave DFT-LDA calculation of the electronic structure and absorption spectrum of copper. Phys. Rev. B 64, 195125 (2001). 0108535.

31. Wooten, F. Optical Properties of Solids (Academic Press, 1972).

32. Harl, J. The Linear Response Function in Density Functional Theory: Optical Spectra and Improved Description of the Electron Correlation. Ph.D. thesis, Universität Wien (2008).

33. Prandini, G. Predicting the Reflectivity and Colour of Metals from First Principles. Ph.D. thesis, EPF Lausanne (2019).

34. Griffiths, D. Introduction to Electrodynamics 3rd edn (Pearson Education, 2007).

35. Schanda, J. Colorimetry: Understanding the CIE System (Wiley-Interscience, 2007).

36. Kajiya, J. T. The rendering equation. SIGGRAPH Comput. Graph. 20, 143-150 (1986).

37. Zunger, A., Wei, S.-H., Ferreira, L. G. \& Bernard, J. E. Special quasirandom structures. Phys. Rev. Lett. 65, 353-356 (1990).
38. Wei, S.-H., Ferreira, L. G., Bernard, J. E. \& Zunger, A. Electronic properties of random alloys: special quasirandom structures. Phys. Rev. B 42, 9622-9649 (1990).

39. Niklasson, G. A., Granqvist, C. G. \& Hunderi, O. Effective medium models for the optical properties of inhomogeneous materials. Appl. Opt. 20, 26-30 (1981).

40. Marini, A., Onida, G. \& Del Sole, R. Quasiparticle electronic structure of copper in the GW approximation. Phys. Rev. Lett. 88, 16403 (2001).

41. Marini, A., Del Sole, R. \& Onida, G. First-principles calculation of the plasmon resonance and of the reflectance spectrum of silver in the $G W$ approximation. Phys. Rev. B 66, 115101 (2002).

42. Rangel, T. et al. Band structure of gold from many-body perturbation theory. Phys. Rev. B 86, 125125 (2012).

43. van Schilfgaarde, M., Kotani, T. \& Faleev, S. Quasiparticle self-consistent GW theory. Phys. Rev. Lett. 96, 226402 (2006).

44. Kotani, T., van Schilfgaarde, M. \& Faleev, S. V. Quasiparticle self-consistent GW method: a basis for the independent-particle approximation. Phys. Rev. B 76 165106 (2007).

45. Heyd, J., Scuseria, G. E. \& Ernzerhof, M. Hybrid functionals based on a screened Coulomb potential. J. Chem. Phys. 118, 8207-8215 (2003).

46. Steinemann, S. G., Anongba, P. N. B. \& Podloucky, R. Color in Pettifor's structure maps: intermetallic compounds for a new use. J. Phase Equilib. 18, 655 (1997).

47. Keast, V. J. et al. The effect of vacancies on the optical properties of $\mathrm{AuAl}_{2}$. J. Phys. Condens. Matter 27, 505501 (2015).

48. Massalski, T., Okamoto, H., Subramanian, P. \& Kacprzak, L. Binary Alloy Phase Diagrams 2nd edn (ASM International, 1990).

49. Rivory, J. Comparative study of the electronic structure of noble-metal-noblemetal alloys by optical spectroscopy. Phys. Rev. B 15, 3119-3135 (1977).

50. Giannozzi, P. et al. QUANTUM ESPRESSO: a modular and open-source software project for quantum simulations of materials. J. Phys. Condens. Matter 21, 395502 (2009).

51. Shirley, E. L. Optimal basis sets for detailed Brillouin-zone integrations. Phys. Rev. B 54, 16464-16469 (1996).

52. Prendergast, D. \& Louie, S. G. Bloch-state-based interpolation: an efficient generalization of the Shirley approach to interpolating electronic structure. Phys. Rev. B 80, 235126 (2009).

53. Prandini, G., Galante, M., Marzari, N. \& Umari, P. SIMPLE code: optical properties with optimal basis functions. Comput. Phys. Commun. 240, 106-119 (2019).

54. Prandini, G., Marrazzo, A., Castelli, I. E., Mounet, N. \& Marzari, N. Precision and efficiency in solid-state pseudopotential calculations. npj Comput. Mater. 4, 72 (2018).

55. Hamann, D. R. Optimized norm-conserving Vanderbilt pseudopotentials. Phys. Rev. B 88, 085117 (2013).

56. Schlipf, M. \& Gygi, F. Optimization algorithm for the generation of ONCV pseudopotentials. Comput. Phys. Commun. 196, 36-44 (2015).

57. van Setten, M. et al. The pseudodojo: training and grading a 85 element optimized norm-conserving pseudopotential table. Comput. Phys. Commun. 226 39-54 (2018).

58. Pizzi, G., Cepellotti, A., Sabatini, R., Marzari, N. \& Kozinsky, B. AiiDA: automated interactive infrastructure and database for computational science. Comput. Mater. Sci. 111, 218-230 (2016).

59. Lejaeghere, K., Van Speybroeck, V., Van Oost, G. \& Cottenier, S. Error estimates for solid-state density-functional theory predictions: an overview by means of the ground-state elemental crystals. Crit. Rev. Solid State Mater. Sci. 39, 1-24 (2014).

60. Lejaeghere, K. et al. Reproducibility in density functional theory calculations of solids. Science 351, aad3000 (2016).

61. Monkhorst, H. J. \& Pack, J. D. Special points for Brillouin-zone integrations. Phys. Rev. B 13, 5188-5192 (1976).

62. Marzari, N., Vanderbilt, D., De Vita, A. \& Payne, M. C. Thermal contraction and disordering of the Al(110) surface. Phys. Rev. Lett. 82, 3296-3299 (1999).

63. van de Walle, A., Asta, M. D. \& Ceder, G. The Alloy Theoretic Automated Toolkit: a user guide. Calphad 26, 539-553 (2002).

64. van de Walle, A. et al. Efficient stochastic generation of special quasirandom structures. Calphad 42, 13-18 (2013).

65. Palik, E. D. Handbook of Optical Constants of Solids. Academic Press Handbook Series (Elsevier Science, 1998).

66. Amiotti, M., Borghesi, A., Guizzetti, G. \& Nava, F. Optical properties of poly crystalline nickel silicides. Phys. Rev. B 42, 8939-8946 (1990).

67. Nishimura, K. et al. Fermi surfaces properties of $\mathrm{AuAl}_{2}, \mathrm{AuGa}_{2}$, and $\mathrm{AuIn}_{2}$ with the $\mathrm{CaF}_{2}$-type cubic structure. Phys. B 536, 588-596 (2018).

68. Furrer, A. Colours in Thin Metallic Films Based on Precious Metals and their Intermetallic Phases. Ph.D. thesis, ETH Zurich (2013).

69. Roberts, E. F. I. \& Clarke, K. M. The colour characteristics of gold alloys. Gold Bull. 12, 9-19 (1979).

70. Yang, H. U. et al. Optical dielectric function of silver. Phys. Rev. B 91, 235137 (2015).

71. Olmon, R. L. et al. Optical dielectric function of gold. Phys. Rev. B 86, 235147 (2012) 


\section{ACKNOWLEDGEMENTS}

We warmly thank Fanny Lalire and Frédéric Diologent for several useful discussions and for sharing with us confidential experimental results. This research was supported by Varinor SA (CH 2800 Delémont, Switzerland).

\section{AUTHOR CONTRIBUTIONS}

N.M. and G.-M. R designed the study; G.P. developed the computational workflow, performed the calculations and wrote the manuscript. All authors discussed and analysed the results and commented on the manuscript.

\section{COMPETING INTERESTS}

The authors declare no competing interests.

\section{ADDITIONAL INFORMATION}

Supplementary information is available for this paper at https://doi.org/10.1038/ s41524-019-0266-0.

Correspondence and requests for materials should be addressed to N.M.
Reprints and permission information is available at http://www.nature.com/ reprints

Publisher's note Springer Nature remains neutral with regard to jurisdictional claims in published maps and institutional affiliations.

(2) Open Access This article is licensed under a Creative Commons Attribution 4.0 International License, which permits use, sharing, adaptation, distribution and reproduction in any medium or format, as long as you give appropriate credit to the original author(s) and the source, provide a link to the Creative Commons license, and indicate if changes were made. The images or other third party material in this article are included in the article's Creative Commons license, unless indicated otherwise in a credit line to the material. If material is not included in the article's Creative Commons license and your intended use is not permitted by statutory regulation or exceeds the permitted use, you will need to obtain permission directly from the copyright holder. To view a copy of this license, visit http://creativecommons. org/licenses/by/4.0/.

(c) The Author(s) 2019 